\title{
Numerical study of the two-replica overlap of the 3D Edwards-Anderson Ising spin glass
}

\author{
Bernd A. Berg, ${ }^{\mathrm{a}, 1}$ Alain Billoire, ${ }^{\mathrm{b}, 2}$ and Wolfhard Janke ${ }^{\mathrm{c}, 3}$ \\ ${ }^{a}$ Dept. of Physics, Florida State University, Tallahassee, FL 32306, USA \\ b Service de Physique Théorique, CEA/Saclay, 91191 Gif-sur-Yvette, France \\ ${ }^{\mathrm{c}}$ Institut für Theoretische Physik, Universität Leipzig, 04109 Leipzig, Germany
}

\begin{abstract}
We present results of recent high-statistics Monte Carlo simulations of the EdwardsAnderson Ising spin-glass model in three dimensions. The study is based on a nonBoltzmann sampling technique, the multi-self-overlap algorithm which is specifically tailored for sampling rare-event states. We thus concentrate on those properties which are difficult to obtain with standard canonical Boltzmann sampling such as the free-energy barriers $F_{B}^{q}$ in the probability densities $P_{J}(q)$ of the Parisi overlap parameter $q$ and the behavior of the tails of the disorder averaged density $P(q)=$ $\left[P_{J}(q)\right]_{\mathrm{av}}$. Our results for the tails disagree with mean-field predictions and support extreme order statistics over many orders of magnitude.
\end{abstract}

Key words: Spin Glass, Edwards-Anderson model, Ising, multicanonical, multi-self-overlap.

\section{Introduction}

A widely studied class of spin-glass materials [1-4] consists of dilute solutions of magnetic transition metal impurities in noble metal hosts, for instance [5] $\mathrm{Au}-2.98 \% \mathrm{Mn}$. In these systems, the interaction between impurity moments is caused by the polarization of the surrounding Fermi sea of the host conduction electrons, leading to an effective interaction of the so-called RKKY form [6]

$$
J_{\mathrm{eff}}(R) \propto \frac{\cos \left(2 k_{F} R\right)}{R^{3}}, \quad k_{F} R \gg 1,
$$

1 Email: berg@hep.fsu.edu

2 Email: billoir@spht.saclay.cea.fr

3 Email: wolfhard.janke@itp.uni-leipzig.de 


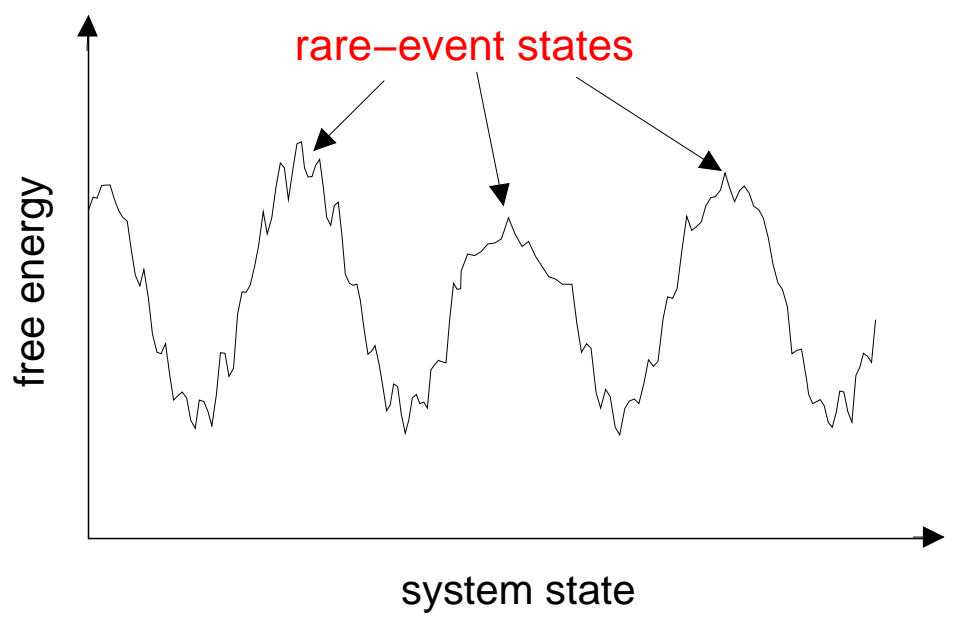

Fig. 1. Typical sketch of the rugged free-energy landscape of spin glasses, with many minima separated by rare-event barriers.

where $k_{F}$ is the Fermi wave number. This constitutes the two basic ingredients necessary for spin-glass behavior, namely

- randomness - in course of the dilution process the positions of the impurity moments are randomly distributed, and

- competing interactions - due to the oscillations in (1) as a function of the distance $R$ between the spins some of the interactions are positive and some are negative.

The competition among the different interactions between the moments means that no single configuration of spins is favored by all of the interactions, a phenomenon which is called "frustration". This leads to a rugged free-energy landscape with probable regions (low free energy) separated by rare-event states (high free energy), illustrated in many previous articles by sketches similar to our Fig. 1. Experimentally this may be inferred from the phenomenon of aging observed in measurements of the remanent magnetization in the spin-glass phase.

Despite the large amount of experimental, theoretical and simulational work done in the past thirty years to elucidate the nature of the spin-glass phase [14], the physical mechanisms underlying its peculiar properties are not yet fully understood. To cope with the complexity of the problem various levels of simplified models have been studied theoretically. A simplified lattice model which reflects the two basic ingredients for spin-glass behavior is the EdwardsAnderson [7] Ising (EAI) model defined through the energy

$$
E=-\sum_{\langle i k\rangle} J_{i k} s_{i} s_{k}
$$


where the fluctuating spins $s_{i}$ can take the values \pm 1 . The coupling constants $J_{i k}$ are quenched random variables taking positive and negative signs, thereby leading to competing interactions. In our study we worked with a bimodal distribution, $J_{i k}= \pm 1$ with equal probabilities. In (2), the lattice sum runs over all nearest-neighbor pairs of a $d$-dimensional (hyper-) cubic lattice of size $N=L^{d}$ with periodic boundary conditions.

A mean-field tractable model, the Sherrington-Kirkpatrick [8] model, emerges when each spin is allowed to interact with all others. Alternatively one may consider the mean-field treatment as an approximation which is expected to become accurate in high dimensions [9]. In physical dimensions, however, its status is still unclear and an alternative droplet model [10] has been proposed. The two treatments yield conflicting predictions. Numerical approaches such as Monte Carlo (MC) simulations can, in principle, provide precise results in physical dimensions. In practice, however, the simulational approach is severely hampered by an extremely slow dynamics of the stochastic process, and the need to consider many disorder realizations.

To overcome the slowing-down problem various ingenious simulation techniques have been devised in the past few years. While some of them only aim at improving the dynamics of the MC process, others are in addition well suited for a quantitative characterization of the free-energy barriers responsible for the slowing-down problem. Among the latter category is the multi-self-overlap (MSO) algorithm [11] which has been employed in our MC simulations [12,13] of the EAI spin-glass model. The purpose of this note is to give an overview of our results in three dimensions.

\section{Model Parameters and Simulation Method}

As order parameter of the EAI model one usually takes the Parisi overlap variable [9]

$$
q=\frac{1}{N} \sum_{i=1}^{N} s_{i}^{(1)} s_{i}^{(2)}
$$

where the spin superscripts label two independent (real) replicas of the same realization of randomly chosen exchange coupling constants $J=\left\{J_{i k}\right\}$. For a given $J$ the probability density of $q$ is denoted by $P_{J}(q)$, and thermodynamic expectation values are computed as

$$
\langle\ldots\rangle_{J} \equiv \sum_{\{s\}}(\ldots) \exp (-\beta H[J]) / \sum_{\{s\}} \exp (-\beta H[J])
$$


where $\beta=1 / T$ is the inverse temperature in natural units. The freezing temperature is known to be at $\beta_{c}=0.90(3)$ [14].

The results depend on the randomly chosen quenched disorder realization, and one must average over many hundreds or even thousands of disorder realizations:

$$
P(q)=\left[P_{J}(q)\right]_{\mathrm{av}}=\frac{1}{\# J} \sum_{J} P_{J}(q), \quad\left[\langle\ldots\rangle_{J}\right]_{\mathrm{av}}=\frac{1}{\# J} \sum_{J}\langle\ldots\rangle_{J}
$$

where $\# J(\rightarrow \infty)$ is the number of realizations considered. Below the freezing temperature, in the infinite-volume limit $N \rightarrow \infty$, a non-vanishing part of $P(q)$ between its two delta-function peaks at $\pm q_{\max }$ characterizes the meanfield picture [9] of spin glasses, whereas in the droplet picture [10] of spin glasses (as well as in ferromagnets) $P(q)$ exhibits only the two delta-function peaks.

For a better understanding of the free-energy barriers sketched in Fig. 1, the probability densities for individual realizations $J$ play the central role. As it is impossible to get complete control over the full state space, and to give a well-defined meaning to the "system state" (the $x$-axis in Fig. 1), one has to concentrate on one or a few characteristic properties. In our work we focused on the order parameter and thus on those free-energy barriers $F_{B}^{q}$ which are reflected by the minima of $P_{J}(q)$. To allow for a visual impression of the encountered shapes, all 640 probability densities $P_{J}(q)$ as obtained in the MSO simulations of the $3 \mathrm{D}$ EAI spin-glass model on a $12^{3}$ lattice at $T=$ $1 \approx 0.88 T_{c}$ have been made available as a Java animation on the Web. Go to www.hep.fsu.edu/ ${ }^{\sim}$ berg, follow the research link to complex systems and from there to the picture show of spin-glass realizations.

Conventional, canonical MC simulations are not suited for a study of such free energy barriers, because the likelihood to generate the corresponding rare-event configurations in the Gibbs canonical ensemble is very small. This problem is overcome by non-Boltzmann sampling with the MSO weight [11]

$$
w_{J}(q)=\exp \left[\beta \sum_{\langle i k\rangle} J_{i k}\left(s_{i}^{(1)} s_{k}^{(1)}+s_{i}^{(2)} s_{k}^{(2)}\right)+S_{J}(q)\right]
$$

where the two replicas are coupled by $S_{J}(q)$ in such a way that a broad MSO histogram $P_{J}^{\mathrm{muq}}(q)$ over the entire accessible range $-1 \leq q \leq 1$ is obtained. When simulating with the MSO weight (6), canonical expectation values of any quantity $O$ can be reconstructed by reweighting, $\langle O\rangle_{J}^{\text {can }}=\left\langle O e^{-S_{J}}\right\rangle_{J} /\left\langle e^{-S_{J}}\right\rangle_{J}$.

For each of the quenched disorder realizations the steps of the MSO algorithm may be summarized as follows: 
- An iterative construction of the weight function $W_{J}(q) \equiv \exp \left(S_{J}(q)\right)$.

- An equilibration period with fixed weight function.

- A production run with fixed weight function.

We measure the dynamics of the MSO algorithm in terms of the autocorrelation time $\tau_{J}^{\text {muq }}$, which is defined by counting the average number of sweeps it takes to complete the cycle $q=0 \rightarrow|q|=1 \rightarrow 0$. Adopting the usual terminology [15] for a first-order phase transition, we shall call such a cycle a "tunneling" event. The weight iteration was stopped after at least 10 "tunneling" events occurred, and in the production runs we collected at least 20 "tunneling" events. To allow for standard reweighting in the temperature we stored besides $P_{J}(q)$ also the time series of $q$, and of the energies and magnetizations of the two replicas. The number of sweeps between measurements was adjusted by an adaptive data compression routine to ensure that each time series consists of $2^{16}=65536$ measurements separated by approximately $\tau_{J}^{\text {muq }}$ sweeps.

\section{Results}

Our simulation temperatures were $T=1 \approx 0.88 T_{c}$ and $T=1.14 \approx T_{c}$. Due to the large number of realizations simulated, the final results are relatively costly. By fitting the averaged autocorrelation times to the power-law ansatz $\ln \left(\left[\tau_{J}^{\mathrm{muq}}\right]_{\mathrm{av}}\right)=a+z \ln (N)$, we obtained $[12] z=2.32(7)$. The quality of the fit is poor and an exponential behavior $\left(\ln \left(\left[\tau_{J}^{\mathrm{muq}}\right]_{\mathrm{av}}\right) \propto N^{\alpha}\right)$ cannot be excluded. It shows that the slowing down is quite off from the theoretical optimum $z=1$, one would expect if the MSO autocorrelation time $\tau_{J}^{\text {muq }}$ was dominated by a random-walk behavior between $q=-1$ and +1 . In multicanonical simulations with broad energy histograms an even larger exponent of $z=2.8(1)$ has been observed [16]. The large values of $z$ suggest that the canonical overlap or energy barriers are not the exclusive cause for the slowing down of spin-glass dynamics below the freezing point. The projection of the multi-dimensional state space onto the $q$ - or $E$-direction direction averages out most of the freeenergy landscape of the model.

\subsection{Free-Energy Barriers $F_{B}^{q}$}

To define effective free-energy barriers $F_{B}^{q}$ we first constructed [12] an auxiliary 1D Metropolis-Markov chain which has the canonical $P_{J}(q)$ probability density as its equilibrium distribution. The tridiagonal transition matrix of this Markov process allows for diagonalization by standard methods. The largest 


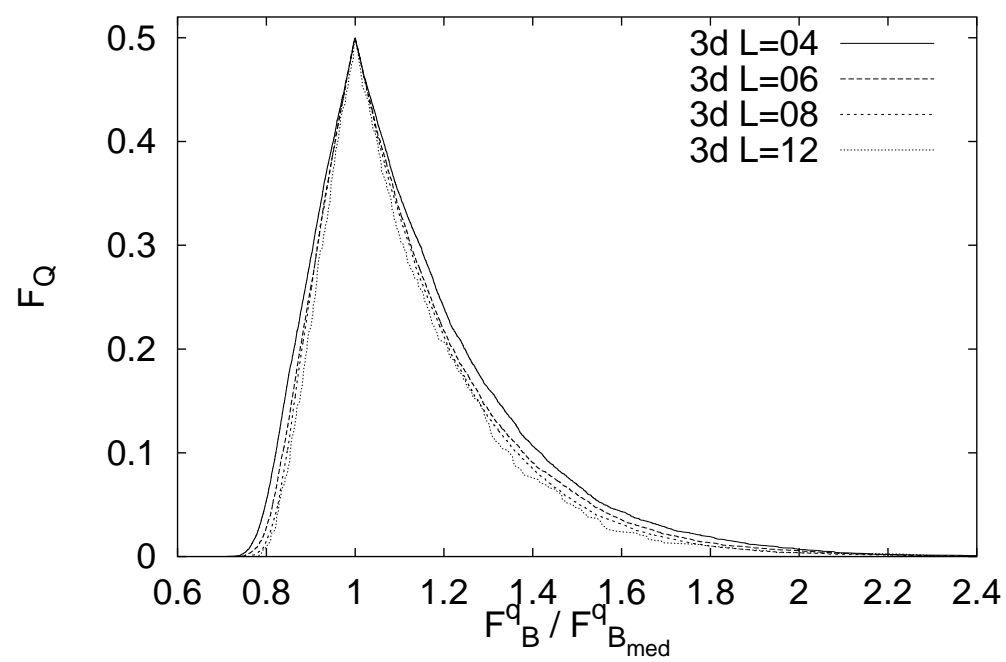

Fig. 2. Peaked distribution function $F_{Q}(9)$ for the overlap barriers (8) in the spinglass phase at $T=1 \mathrm{in}$ units of their median values.

eigenvalue $\lambda_{0}$ equals unity and is non-degenerate. The second largest eigenvalue $\lambda_{1}$ determines the autocorrelation time of the chain. In units of sweeps,

$$
\tau_{B}^{q}=-\frac{1}{N \ln \lambda_{1}} \approx \frac{1}{N\left(1-\lambda_{1}\right)},
$$

which we use to define the associated effective free-energy barrier in the overlap parameter $q$ as

$$
F_{B}^{q} \equiv \ln \left(\tau_{B}^{q}\right)
$$

Our finite-size scaling (FSS) analyses of the thus defined overlap barriers are based on the (cumulative) distribution function $F(x)$. More precisely, we used a peaked distribution function $F_{Q}(x)$ defined [17] by reflecting $F(x)$ at its median value 0.5 ,

$$
F_{Q}(x) \equiv \begin{cases}F(x) & \text { for } F(x) \leq 0.5 \\ 1-F(x) & \text { for } F(x) \geq 0.5\end{cases}
$$

For self-averaging data the function $F_{Q}$ collapses in the infinite-volume limit to $F_{Q}(x)=0.5$ for $x=[x]_{\mathrm{av}}$ and 0 otherwise. For non-self-averaging quantities the width of $F_{Q}$ stays finite. The concept carries over to quantities which diverge in the infinite-volume limit, when for each lattice size scaled variables $x / x_{\text {med }}$ are used, where $x_{\text {med }}$ denotes the median defined through $F\left(x_{\text {med }}\right)=$ $F_{Q}\left(x_{\text {med }}\right)=0.5$. 


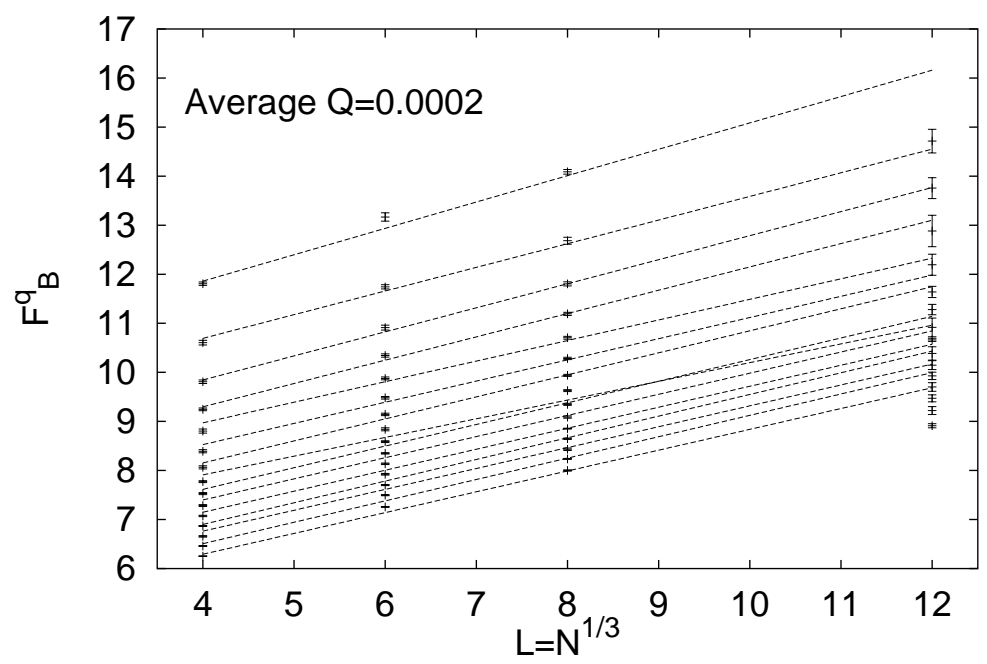

Fig. 3. FSS fits of the overlap barriers $F_{B}^{q}$ in the spin-glass phase at $T=1$ for fixed values of the distribution function, $F=i / 16, i=1, \ldots, 15$ (from bottom to top). Shown are the (unacceptable) results for the mean-field prediction (10).

The behavior of $F_{Q}\left(F_{B}^{q} / F_{B_{\text {med }}}^{q}\right)$ shown in Fig. 2 for $T=1$ clearly suggests that $F_{B}^{q}$ is a non-self-averaging quantity. Non-self-averaging was also observed [12] for the autocorrelation times $\tau_{J}^{\text {muq }}$ of our algorithm while the energy is an example for a self-averaging quantity. For non-self-averaging quantities one has to investigate many samples and should report the FSS behavior for fixed values of the cumulative distribution function $F$. Hence, we performed in Ref. [12] FSS fits for $F=i / 16, i=1, \ldots, 15$, assuming an ansatz suggested by meanfield theory $[18,19]$,

$$
F_{B}^{q}=a_{1}+a_{2} N^{1 / 3}
$$

corresponding to $\tau_{B}^{q} \propto e^{a_{2} N^{1 / 3}}$. The fits are depicted in Fig. 3. The goodnessof-fit parameter $Q$ turned out to be unacceptably small. We therefore also tried fits to the ansatz

$$
F_{B}^{q}=\ln (c)+\alpha \ln (N)
$$

corresponding to $\tau_{B}^{q} \propto N^{\alpha}$. They lead to far better results. As a function of $F$ $(=1 / 16-15 / 16)$ the exponent $\alpha=\alpha(F)$ in the power law (11) varies smoothly from 0.8 to 1.1. A similar analysis [12] for the autocorrelation times $\tau_{J}^{\text {muq }}$ of the MSO algorithm gives exponents $\alpha(F)$ which are larger, $\alpha^{\text {muq }}(F) \approx \alpha_{B}^{q}(F)+1$. This is in agreement with our observation that other relevant barriers exist, which cannot be detected in the overlap parameter $q$. 


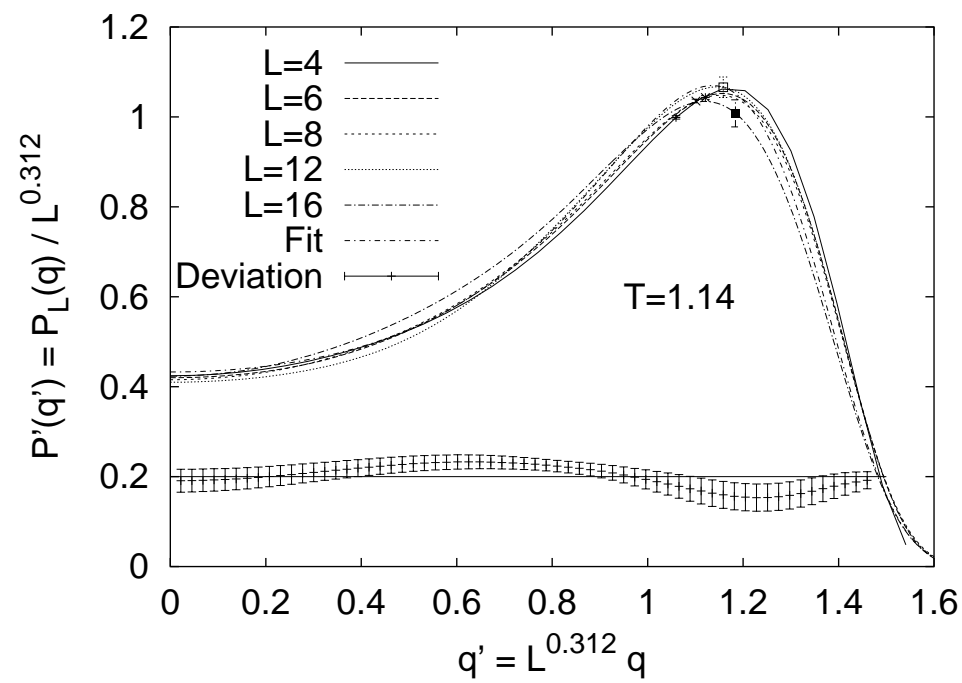

Fig. 4. Rescaled overlap probability densities for the EAI spin-glass model on $L^{3}$ lattices at the critical temperature on a linear scale.

\subsection{Averaged Probability Densities $P(q)$}

At least close to $T_{c}$ one expects that, up to finite-size corrections, the probability densities scale with system size. A method to confirm this visually is to plot $P^{\prime}(q) \equiv \sigma P(q)$ versus $q^{\prime}=q / \sigma$, where $\sigma$ is the standard deviation. By fitting the standard deviation to the expected FSS form $\sigma=c_{1} L^{-\beta / \nu}$ we obtained [13],

$$
\begin{aligned}
& \frac{\beta}{\nu}=0.312(4), Q=0.32 \text { for } T=1.14, \text { and } \\
& \frac{\beta}{\nu}=0.230(4), Q=0.99 \text { for } T=1 .
\end{aligned}
$$

In Fig. 4 we show the scaling plot [13] for $T=1.14$ which demonstrates that the five probability densities collapse onto a single master curve. Remarkably, this still holds at $T=1$ below the critical point.

\subsection{Tails of $P(q)$}

The MSO algorithm becomes particularly powerful when studying the tails of the probability densities which are highly suppressed compared to the peak values, see Fig. 5 which shows $P(q)$ at $T=1.14$ over more than 150 orders of magnitude. Based on the replica mean-field approach, theoretical predictions for the scaling behavior of the tails have been derived by Parisi and collaborators [20]. They showed that $P(q)=P_{\max } f\left(N\left(q-q_{\max }^{\infty}\right)^{x}\right)$ for $q>q_{\max }^{\infty}$ and 


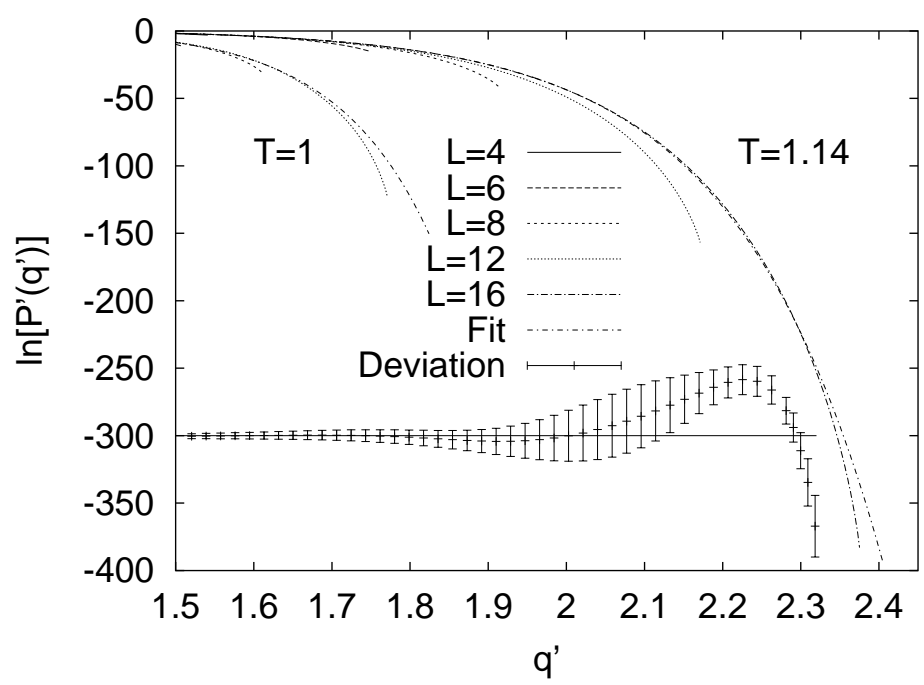

Fig. 5. Tails of the rescaled overlap probability densities for the EAI spin-glass model on $L^{3}$ lattices at the critical temperature on an logarithmic scale. In the lower part of the plots the deviation $P_{16}^{\prime}\left(q^{\prime}\right)-P_{\text {fit }}^{\prime}\left(q^{\prime}\right) \pm \triangle P_{16}^{\prime}\left(q^{\prime}\right)$ of some $L=16$ data from the modified Gumbel fit is shown (with an unimportant offset added in order to be inside the figure).

concluded more quantitatively that

$$
P(q) \sim \exp \left[-c_{1} N\left(q-q_{\max }^{\infty}\right)^{x}\right] \text { for } N\left(q-q_{\max }^{\infty}\right)^{x} \text { large }
$$

with a mean-field exponent of $x=3$. By allowing for an overall normalization factor $c_{0}^{(N)}$ and taking the logarithm twice we have performed fits of the form $[21]$

$$
Y \equiv \ln \left[-\ln \left(P / c_{0}^{(N)}\right)\right]-\ln N=\ln c_{1}+x \ln \left(q-q_{\max }^{\infty}\right)
$$

Consistent fits are only obtained over a rather restricted range of $q$. Using them anyway, and leaving the exponent $x$ as a free parameter, we arrived at the estimate $x=12(2)$, which is much larger than the mean-field value of $x=3$.

By looking for reasonable alternatives we realized that for the 2D $X Y$ model the statistics of extremes has led to a good ansatz with universal properties [22,23]. This is based on a standard result [24,25], due to Fisher and Tippett, Kawata, and Smirnov, for the universal distribution of the first, second, third, ... smallest of a set of $N$ independent identically distributed random numbers. For an appropriate, exponential decay of the random number distribution their probability densities are given by the Gumbel form

$$
f_{a}(x)=C_{a} \exp \left[a\left(x-e^{x}\right)\right],
$$


in the limit of large $N$. The exponent $a$ takes the values $a=1,2,3, \ldots$, corresponding, respectively, to the first, second, third, ... smallest random number of the set, $x$ is a scaling variable which shifts the maximum value of the probability density to zero, and $C_{a}$ is a normalization constant. For certain spin-glass systems the possible relevance of this universal distribution has been pointed out by Bouchaud and Mézard [26]. For the 2D XY model in the spin-wave approximation $[22,23]$ the Gumbel ansatz (16) emerges with a modified value of $a=\pi / 2$.

In our case we set $x=b\left(q^{\prime}-q_{\max }^{\prime}\right)$ and modified the first $x$ on the r.h.s. of (16) to $c \tanh (x / c)$, where $c>0$ is a constant, in order to reproduce the flattening of the densities towards $q^{\prime}=0$. The important large- $x$ behavior of Eq. (16) is not at all affected by this manipulation. By fitting this ansatz to our data we obtained final estimates [13] of $a=0.448$ (40) for $T=1.14$ and $a=0.446$ (37) for $T=1$, respectively. The fits are depicted in Fig. 5 , and for $T=1.14$ our fit is also included in Fig. 4. We see a good consistency between the data and the fit over a remarkably wide range of $q^{\prime}$. Most impressive is the excellent agreement in the tails of the densities. Taking the $T=1.14, L=16$ result at face value, we find a very good description over the remarkable range of $200 / \ln (10) \approx 87$ orders of magnitude.

\section{Summary and Conclusions}

Employing non-Boltzmann sampling with the MSO MC algorithm we have investigated the probability densities $P_{J}(q)$ of the Parisi order parameter $q$. The free-energy barriers $F_{B}^{q}$ as defined in Eq. (8) turn out to be non-self-averaging. The logarithmic scaling ansatz (11) for the barriers at fixed values of their cumulative distribution function $F$ is found to be favored over the mean-field ansatz (10). Further, relevant barriers are still reflected in the autocorrelations of the MSO algorithm.

The averaged densities $P(q)$ exhibit a good FSS collapse onto a $L$-independent master curve at and slightly below the critical temperature. For the scaling of their tails towards $q= \pm 1$ we find no agreement with the decay law predicted by mean-field theory. A good fit over more than 80 orders of magnitude is obtained by using a modified Gumbel ansatz, rooted in extreme order statistics $[24,25]$.

The detailed relationship between the EAI spin-glass model and extreme order statistics remains to be investigated, and it is certainly a challenge to extend the work of Bouchaud and Mézard [26] to the more involved scenarios of the replica theory. In this context, let us remark that we do not find extreme order statistics for the 3D Ising model [27], in contrast to the suggestions of 
Refs. [22,23].

We have performed equilibrium simulations of the model. Using non-equilibrium methods, its critical properties have also been studied [28].

\section{Acknowledgments}

BB would like to thank Prof. C.-K. Hu for inviting him to Taiwan and for the kind hospitality extended to him during his stay.

This work was in part supported by the US Department of Energy under contract DE-FG02-97ER41022. The numerical simulations were performed on the Compaq SC256 computer of CEA in Grenoble under grant p526 and on the T3E computer of NIC in Jülich under grant hmz091.

\section{References}

[1] K. Binder and A.P. Young, Rev. Mod. Phys. 56 (1986) 801.

[2] M. Mézard, G. Parisi, and M.A. Virasoro, Spin Glass Theory and Beyond (World Scientific, Singapore, 1987).

[3] K.H. Fischer and J.A. Hertz, Spin Glasses (University Press, Cambridge, 1991).

[4] A.P. Young (ed.), Spin Glasses and Random Fields (World Scientific, Singapore, 1997).

[5] C.A.M. Mulder, A.J. van Duyneveldt, and J.A. Mydosh, Phys. Rev. B 25 (1982) 515.

[6] M.A. Ruderman and C. Kittel, Phys. Rev. 96 (1954) 99; T. Kasuya, K. Yosida, Phys. Rev. 106 (1957) 893.

[7] S.F. Edwards and P.W. Anderson, J. Phys. F 5 (1975) 965.

[8] D. Sherrington and S. Kirkpatrick, Phys. Rev. Lett. 35 (1975) 1792.

[9] G. Parisi, Phys. Rev. Lett. 43 (1979) 1754.

[10] D.S. Fisher and D.A. Huse, Phys. Rev. B 38 (1988) 386.

[11] B.A. Berg and W. Janke, Phys. Rev. Lett. 80 (1998) 4771.

[12] B.A. Berg, A. Billoire, and W. Janke, Phys. Rev. B 61 (2000) 12143.

[13] B.A. Berg, A. Billoire, and W. Janke, Phys. Rev. E 65 (2002) 045102R.

[14] N. Kawashima and A.P. Young, Phys. Rev. B 53 (1996) R484. 
[15] B.A. Berg and T. Neuhaus, Phys. Rev. Lett. 68 (1992) 9.

[16] B.A. Berg, U.E. Hansmann, and T. Celik, Phys. Rev. B 50 (1994) 16444.

[17] B.A. Berg, Introduction to Monte Carlo Simulations and Their Statistical Analysis, in preparation.

[18] N.D. Mackenzie and A.P. Young, Phys. Rev. Lett. 49 (1982) 301; J. Phys. C 16 (1983) 5321; K. Nemoto, J. Phys. A 21 (1988) L287; G.J. Rodgers and M.A. Moore, J. Phys. A 22 (1989) 1085; D. Vertechi and M.A. Virasoro, J. Phys. (France) $\mathbf{5 0}$ (1989) 2325.

[19] R.N. Bhatt, S.G.W. Colborne, M.A. Moore, and A.P. Young, unpublished, as quoted by Rodgers and Moore (Ref. [18]); see also S.G.W. Colborne, J. Phys. A 23 (1990) 4013; H. Kinzelbach and H. Horner, Z. Phys. B 84 (1991) 95; A. Billoire and E. Marinari, J. Phys. A 34 (2001) L727.

[20] S. Franz, G. Parisi, and M.A. Virasoro, J. Phys. I (France) 2 (1992) 1869; G. Parisi, F. Ritort, and F. Slanina, J. Phys. A 26 (1993) 3775; J.C. Ciria, G. Parisi, and F. Ritort, J. Phys. A 26 (1993) 6731.

[21] W. Janke, B.A. Berg, and A. Billoire, in: Non-Perturbative Methods and Lattice $Q C D$, Proceedings of the International Workshop, Zhongshan University, Guangzhou (Canton), China, May 15-20, 2000, eds. X.-Q. Luo and E.B. Gregory (World Scientific, Singapore, 2001), p. 242.

[22] S.T. Bramwell, K. Christensen, J.-Y. Fortin, P.C.W. Holdsworth, H.J. Jensen, S. Lise, J.M. López, M. Nicodemi, J.-F. Pinton, and M. Sellitto, Phys. Rev. Lett. 84 (2000) 3744.

[23] S.T. Bramwell, J.-Y. Fortin, P.C.W. Holdsworth, S. Peysson, J.-F. Pinton, B. Portelli, and M. Sellitto, Phys. Rev. E 63 (2001) 041106.

[24] E.J. Gumbel, Statistics of Extremes (Columbia University Press, New York, 1958).

[25] J. Galambos, The Asymptotic Theory of Extreme Order Statistics, 2nd Edition (Krieger Publishing, Malabar, Florida, 1987).

[26] J.-P. Bouchaud and M. Mézard, J. Phys. A 30 (1997) 7997.

[27] B.A. Berg, A. Billoire, and W. Janke, cond-mat/0205377, submitted to Phys. Rev. E.

[28] Y. Ozeki and N. Ito, Phys. Rev. B 64 (2001) 024416. 